\title{
WILEY-VCH
}

DOI: $10.1002 /$ ((please add manuscript number))

Article type: Full Paper

\section{Magnetometry of Individual Polycrystalline Ferromagnetic Nanowires}

Naveen Shamsudhin", Ye Tao, Jordi Sort, Bumjin Jang, Christian L. Degen, Bradley J. Nelson and Salvador Pané

Naveen Shamsudhin, Bumjin Jang, Prof. Bradley J. Nelson and Dr. Salvador Pané

Multi-Scale Robotics Laboratory, ETH Zurich, 8092 Zurich, Switzerland

E-mail: snaveen@ethz.ch

Dr. Ye Tao and Prof. Christian L. Degen

Department of Physics, ETH Zurich, 8093 Zurich, Switzerland

Prof. Jordi Sort

Institució Catalana de Recerca i Estudis Avançats (ICREA) and Departament de Física, Universitat Autònoma de Barcelona, 08193 Bellaterra, Barcelona, Spain

Keywords: cantilever magnetometry, ferromagnetic nanowires, nanorobotics, electron backscattered diffraction

Ferromagnetic nanowires are finding use as untethered sensors and actuators for probing micro- and nanoscale biophysical phenomena, such as for localized sensing and application of forces and torques on biological samples, for tissue heating through magnetic hyperthermia, and for micro-rheology. Quantifying the magnetic properties of individual isolated nanowires is crucial for such applications. We use dynamic cantilever magnetometry to measure the magnetic properties of individual sub-500nm diameter polycrystalline nanowires of $\mathrm{Ni}$ and $\mathrm{Ni}_{80} \mathrm{Co}_{20}$ fabricated by template-assisted electrochemical deposition. The values are compared with bulk, ensemble measurements when the nanowires are still embedded within their growth matrix. We find that single-particle and ensemble measurements of nanowires yield significantly different results that reflect inter-nanowire interactions and chemical modifications of the sample during the release process from the growth matrix. The results highlight the importance of performing single-particle characterization for objects that will be used as individual magnetic nanoactuators or nanosensors in biomedical applications. 


\section{WILEY-VCH}

\section{Introduction}

Magnetic nanostructures are ideal platforms for transducing external control signals to target sites deep within biological tissues. Physical forces and torques exerted on these magnetic agents by spatio-temporally designed magnetic fields are used for their locomotion and site-targeted localization in bodily fluids ${ }^{[1-3]}$. Body-endogenous and exogenous magnetic nanoparticles have enabled on-demand in vivo triggering of localized hyperthermia ${ }^{[4]}$, neuronal stimulation ${ }^{[5,6]}$ and activation of cellular signaling pathways ${ }^{[7,8]}$. In vitro, they are used as wireless actuators to exert and measure forces and torques on single molecular systems $^{[9]}$, and on individual cells to investigate their mechano-responsive behavior ${ }^{[10-12]}$. Additionally, they are increasingly used as mobile sensors for probing local microrheological properties $^{[13,14]}$. The application of nanomagnetic components in structures with fluidic mobility, incorporating sensing, actuation and advanced on-demand functionalities is known as magnetic nanorobotics ${ }^{[15]}$.

While the majority of current magnetic particle-based biophysical assays have used $\mu \mathrm{m}$ and sub- $\mu \mathrm{m}$ sized spherical superparamagnetic, paramagnetic and ferromagnetic particles, magnetic nanostructures in a variety of geometries like helices, coils, solid wires and chains of beads have been investigated for their enhanced mobility and maneuverability in fluids ${ }^{[16-}$ ${ }^{19]}$. An important class of magnetic nanostructures for these applications is solid ferromagnetic nanowires, with sub-500 $\mathrm{nm}$ diameter and sub-50 $\mu \mathrm{m}$ length, as they are optically visible in vitro and allow for generation of a large magnetic force per unit volume compared to commercial magnetic particles. In addition, their large geometric aspect ratio tailors their magnetic anisotropy allowing for application of torques and wrenching motion on tethered cells and molecules. They have also demonstrated enhanced hyperthermia effect $^{[20,21]}$ compared to superparamagnetic iron oxide nanoparticles (SPIONS) ${ }^{[4]}$.

The quantitative measurement of the magnetic properties of individual isolated nanowires, including the magnetic moment, saturation magnetization, remanence, coercive field and saturation field, is crucial if they are to be used as quantitative force-torque actuators and sensors for mechanobiology or microrheology, as the torque and force exerted by an external magnetic field or gradient on a free floating or biologically tethered structure is directly related to the total magnetic moment by $\tau=m(B) \times B$ and $F=m(B) \cdot \nabla B$ respectively. Furthermore, the magnetic hysteresis loop of single nanowires is a direct measure of the heat- 


\section{WILEY-VCH}

generation capability for magnetic hyperthermia. The quantification of magnetic properties of individual nanowires by either experimental or computational means is difficult. Experimentally, the flux from an individual nanowire is orders of magnitude smaller than the noise level of commercial instruments like the vibrating sample magnetometer (VSM) or the alternate gradient magnetomer (AGM). Computationally, the overall objects' dimensions approach the material grain-sizes, causing bulk-magnetic modeling assumptions of grain orientation and randomization to become invalid ${ }^{[22,23]}$. The objects are also typically too large for a strict single-domain assumption to hold at low fields.

We use dynamic cantilever magnetometry $(\mathrm{DCM})^{[24]}$ to analyze individual, electrochemically grown ferromagnetic transition metal and alloy nanowires. We determine the saturation magnetization, remanence, coercive fields, saturating fields of electrodeposited polycrystalline nanowires composed of $\mathrm{Ni}$ and $\mathrm{Ni}_{80} \mathrm{Co}_{20}$. As a method for nanowire production, electrodeposition offers the widest material generality, geometric tenability, scalability, and multi-material hybrid compatibility ${ }^{[25]}$. A change of template size offers direct geometry-tunability from several tens of nanometers to millimeters, spanning a variety of biological lengths scales. As a method for quantification, cantilever magnetometry has the important advantage of providing direct and quantitative access to the magnetic moment of the sample. This is in contrast to other sensitive magnetic measurement techniques like single nitrogen-vacancy magnetometry ${ }^{[26]}$, superconducting quantum interference device (SQUID) magnetometry ${ }^{[27]}$, scanning hall probe microscopy and magnetic force microscopy (MFM), that all measure stray fields external to, and often at an undefined distance from, a sample. Anisotropic magneto-resistance (AMR) measurements and Magneto-optical Kerr effect (MOKE) have also been used to uncover reversal mechanisms in individual nanowires, but they do not measure the magnetic moment crucial for quantitative force/torque applications. In addition, cantilever magnetometry can be operated over a wide range of temperatures, including room temperature. DCM has been successfully applied to sputtered and evaporated thin films ${ }^{[28]}$, chemical vapor deposited Fe-filled $\mathrm{CNTs}^{[29]}$, atomic layer deposited (ALD) Ni nanotubes $^{[30]}$, magnetron-sputtered amorphous $\mathrm{CoFeB}$ nanotubes ${ }^{[31]}$ and evaporated $\mathrm{Ni}$ and Co nanolines ${ }^{[24,32]}$.

Our nanowires are electrochemically grown by pulse-plating ${ }^{[25]}$ in commercial aluminum oxide (AAO) templates (see SI and Figure S1). After template etching, the nanowires are released into ethanol/water, and a drop of nanowire suspension is spotted on a glass substrate. 


\section{WILEY-VCH}

The nanowires are then picked up and mounted onto the tips of custom ultrasensitive silicon cantilevers (Figure 1) using an optical micromanipulation system and fixed using a small dab of epoxy glue. The spring constants of the cantilevers are in the ranges of $80-150 \mu \mathrm{N} / \mathrm{m}$ and typical mechanical quality factors ( $\mathrm{Q}$ factors) of the cantilevers at zero field and $4 \mathrm{~K}$ are in the range of 20,000-40,000. When better sensitivity is needed, Q can be increased to 200,000 with proper surface passivation of the cantilever ${ }^{[33]}$. The beam deflection is monitored using an optical interferometer. Measurements are conducted at $4 \mathrm{~K}$ within a high vacuum environment. The cantilever resonant frequency change, resulting from the torque induced by the magnetic moment, is tracked under a magnetic field sweep. At large applied fields, the frequency shift can be modeled by employing a Stoner-Wohlfarth (SW) uniformly magnetized particle approximation, given in S.I units, ${ }^{[24,34]}$,

$$
\frac{\Delta f}{f_{0}}=\frac{\mu_{0} m_{s} H H_{k}}{2 k_{0} L_{e}^{2}\left(H+H_{k}\right)}
$$

where $f_{0}$ is the zero-field frequency, $m_{s}$ is the saturation magnetic moment, $k_{0}$ is the cantilever spring constant, $L_{e}$ is the effective cantilever length, and $H_{k}$ is the effective uniaxial anisotropy field. The effective anisotropy may include magnetostatic, magnetocrystalline and magnetoelastic contributions of the sample under investigation. All the measurements are performed at zero-field cooled condition (ZFC). After the magnetometry measurements, the cantilevers are transferred to a scanning electron microscope (SEM) equipped with electron backscatter diffraction (EBSD) and energy-dispersive X-ray spectroscopy (EDX) detectors for determining the morphology, crystallinity and chemical composition of the nanowires.

\section{Results and Discussion}

Figure 2 shows the frequency response of an electrochemically grown $\mathrm{Ni}$ nanowire (diameter $d=378 \mathrm{~nm}$, length $l=4.5 \mu \mathrm{m}$ ), whose long axis is aligned along the cantilever axis as schematically shown in Fig. 1a. The cantilever oscillates in the xz plane while the magnetic field is swept along the z-axis. This measurement configuration (axial magnetization) is important from a nanorobotic standpoint where individual nanowires are mobile in liquid and can physically rotate and align along their long axis (magnetic easy axis) under an applied magnetic field. The axial magnetization behavior determines the force and torque capabilities of the nanowire when used as a nanoactuator or sensor. The $\Delta f(H)$ curve of the Ni nanowire in this axial configuration is cusp-like with an increase in frequency shift as the field is increased. The curve exhibits high-field $\left(\mu_{0} H>0.1 \mathrm{~T}\right)$ reversibility with an 
asymptotic behavior, while hysteresis is present at low fields $\left(\mu_{0} H<0.1 \mathrm{~T}\right)$. Two large jumps in the frequency response are observed at $\mu_{0} H=28.4 \mathrm{mT}$ and $\mu_{0} H=-25.3 \mathrm{mT}$. This lowfield discontinuity, where the frequent shift changes sign from negative to positive, is defined as the switching field $\left(H_{s w_{+}}, H_{s w_{-}}\right)$.

The frequency response at high fields, beyond $H_{s w}$, is fit to Equation 1 with $m_{s}$ and $H_{k}$ as fit parameters. The estimated saturation magnetic moment $m_{s}=1.547 \pm 0.15 \times 10^{-13} \mathrm{Am}^{2}$ (mean $\pm \mathrm{SD}$ ) corresponds to an order of $10^{10} \mu_{B}$ (Bohr magnetons) and the effective anisotropy field is $\mu_{0} H_{k}=0.328 \pm 0.33 \mathrm{~T}$. The saturation magnetization $M_{s}=m_{s} / V$ can then be calculated as $\mu_{0} M_{S}=0.384 \pm 0.09 \mathrm{~T}$. The error in volume of the nanowire estimated from post-magnetometry SEM contributes to the uncertainty in $M_{S}$. To determine the field dependence of the volume averaged magnetization $M$, the above model can be adapted according to Buchter et al. ${ }^{[35]}$. In the axial magnetization configuration, the DCM frequency shift at low fields is proportional to the effective magnetization in the $\mathrm{z}$-direction even in the presence of possible non-uniform spatial distribution of magnetization within the wire ${ }^{[31]}$. The $M(H)$ loop thus obtained exhibits a bistable hysteresis behavior (Figure 2c) with large remanent saturation magnetization $M_{r} \sim 0.87 M_{s}$. After magnetic switching, the Ni nanowire reaches a reversed magnetization state with $M\left(H_{s w}+\delta\right) \sim 0.80 M_{s}$. The coercive field can be defined as $\mu_{0}\left(H_{s w+}-H_{s w_{-}}\right) / 2$ and equals $27 \mathrm{mT}$. The slight asymmetry in the switching fields may arise due to an exchange-coupled nickel oxide surface layer ${ }^{[35]}$. The divergence of the $M(H)$ curve near zero field is due to an artifact in division by a very small number. Another extrinsic magnetic property of interest in magnetic nanorobotics is the saturating field $H_{s}$, defined as the field at which the magnetization reaches $95 \%$ of saturation value, which is $70 \mathrm{mT}$.

The axial magnetization behavior of the single nanowire measured by cantilever magnetometry contrasts with the ensemble magnetic measurement of the Ni nanowire array embedded in AAO template measured with a vibrating sample magnetometer (VSM) (Figure 3). The bistable magnetic behavior of the single nanowire is absent in the ensemble magnetic loop, which is a direct consequence of the magnetostatic self-interaction of the nanowire array. The close packing of the wires in the array makes it harder to magnetize them $\left(H_{S} \sim 250 \mathrm{mT}\right)$ and furthermore reduces their saturation remanence to a low value $\left(M_{r} \sim 0.1 M_{S}\right)$ as is evident in the extreme loop shear. Analytical models have been developed to model the effective dipolar interaction field to quantify the behavior of non-interacting 


\section{WILEY-VCH}

wires ${ }^{[36,37]}$. Parameterization of such models is difficult owing to the variation distribution in pore-size, inter-pore distance, filling fraction and lengths of nanowires in the membrane (see Figure S1 for SEM images of nanowires embedded in template membrane). The large $M_{r}$ in the single nanowire measurement reveals that most of the spins are aligned along the long axis of the wire even after removal of the external field. The energy minimization of this magnetic spin configuration comes from the fact that the ends of the nanowire, defect locations and geometric irregularities, harbor non-uniform magnetic states, such as vortices and closure domains, to minimize the total stray field ${ }^{[38,39]}$.

Electrodeposition offers the possibility to develop magnetic nanostructures out of alloys and intermetallics. The nickel-cobalt ( $\mathrm{NiCo}$ ) alloy system is interesting because the overall magnetic anisotropy can be tuned not only via the nanowire geometry but also by utilizing the variable magnetocrystalline anisotropy across the alloy composition. Low to high coercivity can be obtained using Ni-rich (fcc-phase), equal Co/Ni stoichiometry (fcc-hcp mixed phases) and Co-rich (hep-phase) alloys respectively ${ }^{[0,41]}$. DCM is used to probe the magnetic properties of individual electrodeposited $\mathrm{Ni}_{80} \mathrm{Co}_{20}$ solid nanowires. DCM is performed on three wire samples of increasing aspect ratio $\left(\mathrm{NW}_{1}: d=309 \mathrm{~nm} l=3.0 \mu \mathrm{m}, \mathrm{NW}_{2}: d=\right.$ $345 \mathrm{~nm} l=7.2 \mu \mathrm{m}, \mathrm{NW}_{3}: d=358 \mathrm{~nm} l=13.8 \mu \mathrm{m}$, for SEM images, see Figure S2).

The axial magnetization $\Delta f(H)$ curves of all three $\mathrm{Ni}_{80} \mathrm{Co}_{20}$ nanowires exhibit qualitatively the same behavior as the Ni nanowire, with high-field reversibility and asymptoticity, and a pronounced low-field switching event (Figure 4a,b). The increased magnetic moment of the longer wires results in a relative increase in frequency shift of the measurement cantilever. The coercive field of the NiCo wires is in the range of $\mu_{0} H_{c} \sim 14-18 \mathrm{mT}$. An example of a $M(H)$ loop of $\mathrm{NW}_{3}$ is shown in Figure 4c. The extrinsic magnetic properties of all three NiCo wires are tabulated in Table 1. Analogous to the nickel nanowire, the NiCo wires exhibit a $M_{r}$ between $87-95 \%$ of $M_{S}$. Approximately $50-100 \mathrm{mT}$ is required for an individual NiCo nanowire to reach saturation. No direct dependence on the geometric-aspect ratio was observed on $H_{c}, H_{s}$ or on $M_{r}$.

After magnetometry, the cantilever-bound nanowires were transferred to an SEM for morphology and volumetric analysis followed by EBSD to determine the crystalline structure and orientation (Figure 5). The crystallinity was probed using an EBSD raster step size of $10 \mathrm{~nm} 10 \mathrm{~nm}$ with a beam acceleration voltage of $20 \mathrm{kV}$. The diffraction pattern of $\mathrm{Ni}$ and 


\section{WILEY-VCH}

$\mathrm{Ni}_{80} \mathrm{Co}_{20}$ matched a FCC index, with crystallite sizes ranging from $10-100 \mathrm{~s}$ of $\mathrm{nm}$. Substantial crystal twinning was observed in the Ni nanowire, with grain sizes relatively larger than those of $\mathrm{Ni}_{80} \mathrm{Co}_{20}$. The EBSD map for a representative $\mathrm{Ni}_{80} \mathrm{Co}_{20}$ wire, obtained from the same fabrication batch, was made across its length, and is illustrated for two locations, namely $R_{1}$ and $R_{2}$, in Figure 5 b. EDX spot-mapping at end locations $R_{1}$ and $R_{3}$ revealed a chemical composition of approximately $80 \%$ nickel and $20 \%$ cobalt (see Figure S4). This confirms the uniformity of alloy composition during electrochemical growth and across the length of the nanowire. The EBSD pole figures reveal that there is no preferential orientation for the crystallites and that they are randomly distributed for both material systems. The symmetric FCC structure coupled to the random crystal orientation diminishes the contribution of magnetocrystalline anisotropy to the overall magnetic behavior of the nanowire.

The coercive field of all three $\mathrm{Ni}_{80} \mathrm{Co}_{20}$ nanowires, as observed by DCM, is about $50 \%$ of the coercive field of the Ni nanowire. This reduction in coercivity by one half is also observed in the ensemble VSM measurements of the nanowire array (Table 1 and Figure S3). This clearly establishes the magnetic softening of electrochemically grown $\mathrm{Ni}_{80} \mathrm{Co}_{20}$ nanowires as compared to $\mathrm{Ni}$, as was previously reported in the case of electrodeposited thin films ${ }^{[42]}$. The large wire diameters $(300-380 \mathrm{~nm}$, which are much larger than the magnetic coherence length), the polycrystallinity and the surface roughness of the wires are suggestive of a magnetization reversal via defect-localized nucleation and domain wall propagation ${ }^{[27,43,44]}$. The SEM images reveal surface roughness and branching along the length of the wire (Figure S2). These can serve as nucleation sites for domains with reversed magnetization, due to enlarged stray fields at these points. Electrochemically grown large-diameter polycrystalline nanowires are morphologically quite rough compared to other smaller diameter and smooth elongated nanostructures grown by magnetron sputtering or CVD which magnetically reverse in a well-predictable manner ${ }^{[29,31]}$. The smaller frequency switching events (Barkhausen-like jumps) observed in Figure $4 \mathrm{~b}$ (inset) for $\mathrm{Ni}_{80} \mathrm{Co}_{20} \mathrm{NW}_{2}$ are indicative of domain wall nucleation, pinning and depinning events ${ }^{[45]}$. We note that the measured coercivity did not exhibit aspect-ratio dependence.

The low temperature bulk crystalline values of saturation magnetization $\left(\mu_{0} M_{S}\right)$ is $0.510 \mathrm{~T}$ for $\mathrm{Ni}^{[46]}$, and $0.697 \mathrm{~T}$ for $\mathrm{Ni}_{80} \mathrm{Co}_{20}$ as established by the Slater-Pauling curve ${ }^{[47]}$ which dictates a linear increase in $M_{S}$ for increasing Co content in NiCo alloys. On the contrary 


\section{WILEY-VCH}

DCM estimates of average saturation magnetization (Table 1) are lower than these bulk crystalline values. Several reasons may be attributed to these reduced estimates. The first is the unknown volume of surface and internal oxides. The geometrical volume of the nanowires estimated from SEM images does not yield information on the volume contribution of oxides. The wet electrochemical deposition process can lead to oxygen inclusion and formation of internal oxides ${ }^{[48]}$. Furthermore stochastic oxidation during $\mathrm{NaOH}$-based template etching, and during subsequent storage of the free wires in ethanol/ $\mathrm{H}_{2} \mathrm{O}$ solvent, and room temperature drying before cantilever magnetometry, can lead to formation of surface oxides such as $\mathrm{NiO}$, $\mathrm{CoO}$ or $\mathrm{Co}_{2} \mathrm{O}_{3}$. Previous measurements on nickel nanotubes and nanolines by cantilever magnetometry have also revealed a considerable variation in estimates of saturation magnetization, ranging from $0.376-0.820 \mathrm{~T}^{[30,32,35]}$, mainly arising from volume uncertainty. Recent studies have measured a substantial reduction in the relative bulk magnetic moment when ferromagnetic ( $\mathrm{Ni}$ and $\mathrm{Co}$ ) nanowires and commercial magnetic particles where kept in biological solvents ${ }^{[49,50]}$. The post-synthesis process of template removal and release process can also lead to mechanical damage and breakage of the wires promoting crack-driven oxidation and corrosion.

In magnetic nanowire applications in mechanobiology ${ }^{[10]}$ or in the microrheological characterization of complex bio-fluids ${ }^{[14]}$, the application of the maximum achievable torques and forces is desirable. This can be achieved by saturating the magnetic moment of the nanowire. The saturation fields of individual $\mathrm{Ni}$ and NiCo wires measured by DCM lie in between $53-118 \mathrm{mT}$, while the values obtained for the wire ensembles from VSM measurements exceed $250 \mathrm{mT}$. The DCM measurements indicate that external magnetic field generation systems need not exceed fields of $120 \mathrm{mT}$ to saturate the nanowires. Depending on the operational volume and the field-gradient complexity required for the particular in vitro or in vivo application, the design of such magnetic field generation and manipulation systems can be a challenging task ${ }^{[51-53]}$. It is quite often the case that theoretical bulk magnetic properties or ensemble measurement data is used for modeling and data interpretation ${ }^{[54]}$, as the measurement of the field dependence of the magnetic moment of individual nanostructures is difficult. We quantified the lowering and the stochastic oxidation dependent uncertainty in the saturation magnetization of these nanowires. These measurements pave the way for use of these and other nanomagnetic materials in quantitative studies in mechanotransduction and microrheology as the uncertainties associated with the magnetic moment directly propagate into the forces and torques applied or measured. Finally, the 


\section{WILEY-VCH}

quantification of the large magnetic remanence and the estimation of the coercive field, hint the use of pre-magnetized nanowires in sensor-actuator applications.

\section{Conclusion}

In summary, we investigated the magnetic properties of individual electrochemically-grown polycrystalline $\mathrm{Ni}$ and $\mathrm{Ni}_{80} \mathrm{Co}_{20}$ nanowires. The increased softness and the comparable saturation magnetization gives $\mathrm{Ni}_{80} \mathrm{Co}_{20}$ nanowires an advantage over $\mathrm{Ni}$ nanowires for nanoactuator applications as they produce a higher force-torque per unit magnetic field. Dynamic cantilever magnetometry is an excellent tool for quantitative magnetic characterization of individual mesoscopic structures in a transition range between bulk and single domain and in the presence of fabrication-induced stochastic polycrystal fine-structure formation and oxidation. DCM can provide invaluable feedback to the model-driven synthesis of advanced magnetic particles and design of magnetic manipulation systems for biomedical applications.

\section{Experimental Section}

The fabrication details of the nanowires can be found in the supporting information.

\section{Supporting Information}

Supporting Information is available from the Wiley Online Library or from the author.

\section{Acknowledgements}

Naveen Shamsudhin and Ye Tao contributed equally to this work. The authors would like to thank Karsten Kunze for EBSD measurements, Xiangzhong Chen, Ann Hirt, Andrew Petruska, Olgaç Ergeneman, Daniel Ahmed and Abu Sebastian for helpful discussions. N.S and S.P acknowledge funding from the European Research Commission (ERC ICT FETOpen) under the MANAQA Project, Grant No 296679. J.S and B.J acknowledge partial financial support from Generalitat de Catalunya (2014 SGR-1015 project) and Swiss National Science Foundation (Interdisciplinary Project Grant 147152) respectively. C.D acknowledges funding from the ERC through Starting Grant 309301.

Received: ((will be filled in by the editorial staff))

Revised: ((will be filled in by the editorial staff)) Published online: ((will be filled in by the editorial staff))

[1] A. S. Lübbe, C. Bergemann, H. Riess, F. Schriever, P. Reichardt, K. Possinger, M. Matthias, B. Dörken, F. Herrmann, R. Gürtler, P. Hohenberger, N. Haas, R. Sohr, B. 


\section{WILEY-VCH}

Sander, a J. Lemke, D. Ohlendorf, W. Huhnt, D. Huhn, Cancer Res. 1996, 56, 4686.

[2] A. Servant, F. Qiu, M. Mazza, K. Kostarelos, B. J. Nelson, Adv. Mater. 2015, 1.

[3] S. Martel, J.-B. Mathieu, O. Felfoul, A. Chanu, E. Aboussouan, S. Tamaz, P.

Pouponneau, L. Yahia, G. Beaudoin, G. Soulez, M. Mankiewicz, Appl. Phys. Lett. 2007, 90, 114105 .

[4] K. Maier-Hauff, F. Ulrich, D. Nestler, H. Niehoff, P. Wust, B. Thiesen, H. Orawa, V. Budach, A. Jordan, J. Neurooncol. 2011, 103, 317.

[5] R. Guduru, P. Liang, J. Hong, A. Rodzinski, A. Hadjikhani, J. Horstmyer, E. Levister, S. Khizroev, Nanomedicine 2015, 10, 2051.

[6] R. Chen, G. Romero, M. G. Christiansen, A. Mohr, P. Anikeeva, Science 2015, 347, 1477.

[7] X. Long, J. Ye, D. Zhao, S.-J. Zhang, Sci. Bull. 2015, DOI 10.1007/s11434-015-0902-0.

[8] S. a. Stanley, J. Sauer, R. S. Kane, J. S. Dordick, J. M. Friedman, Nat. Med. 2015, 21, 92.

[9] I. De Vlaminck, C. Dekker, Annu. Rev. Biophys. 2012, 41, 453.

[10] N. J. Sniadecki, A. Anguelouch, M. T. Yang, C. M. Lamb, Z. Liu, S. B. Kirschner, Y. Liu, D. H. Reich, C. S. Chen, Proc. Natl. Acad. Sci. U. S. A. 2007, 104, 14553.

[11] G. F. Weber, M. A. Bjerke, D. W. DeSimone, Dev. Cell 2012, 22, 104.

[12] P. Tseng, J. W. Judy, D. Di Carlo, Nat. Methods 2012, 9, DOI 10.1038/nmeth.2210.

[13] L. Chevry, N. K. Sampathkumar, a. Cebers, J. F. Berret, Phys. Rev. E - Stat. Nonlinear, Soft Matter Phys. 2013, 88, 1.

[14] J.-F. Berret, Nat. Commun. 2016, 7, 10134.

[15] S. Martel, J. Nanoparticle Res. 2015, 17, DOI 10.1007/s11051-014-2734-2.

[16] S. Tottori, L. Zhang, F. Qiu, K. K. Krawczyk, A. Franco-Obregõn, B. J. Nelson, Adv. Mater. 2012, 24, 811.

[17] E. J. Smith, D. Makarov, S. Sanchez, V. M. Fomin, O. G. Schmidt, Phys. Rev. Lett. 


\section{WILEY-VCH}

2011, 107, DOI 10.1103/PhysRevLett.107.097204.

[18] R. Dreyfus, J. Baudry, M. L. Roper, M. Fermigier, H. a. Stone, J. Bibette, Nature 2005, $437,862$.

[19] T. Petit, L. Zhang, K. E. Peyer, B. E. Kratochvil, B. J. Nelson, Nano Lett. 2012, 12, 156.

[20] J. Alonso, H. Khurshid, V. Sankar, Z. Nemati, M. H. Phan, E. Garayo, J. a. García, H. Srikanth, J. Appl. Phys. 2015, 117, 17D113.

[21] D. S. Choi, J. Park, S. Kim, D. H. Gracias, M. K. Cho, Y. K. Kim, a Fung, S. E. Lee, Y. Chen, S. Khanal, S. Baral, J. H. Kim, J. Nanosci. Nanotechnol. 2008, 8, 2323.

[22] J. J. Abbott, O. Ergeneman, M. P. Kummer, A. M. Hirt, B. J. Nelson, IEEE Trans. Robot. 2007, 23, 1247.

[23] G. Herzer, Acta Mater. 2013, 61, 718.

[24] B. C. Stipe, H. J. Mamin, T. D. Stowe, T. W. Kenny, D. Rugar, Phys. Rev. Lett. 2001, $86,2874$.

[25] B. Özkale, N. Shamsudhin, G. Chatzipirpiridis, M. Hoop, F. Gramm, X.-Z. Chen, X. Martí, J. Sort, E. Pellicer, S. Pané, ACS Appl. Mater. Interfaces 2015, 150317082250001.

[26] A. Dussaux, P. Schoenherr, K. Chang, N. Kanazawa, Y. Tokura, C. L. Degen, D. Meier, Arxiv Prepr. 2015, 1503.06622.

[27] W. Wernsdorfer, B. Doudin, D. Mailly, K. Hasselbach, A. Benoit, J. Meier, J.-P. Ansermet, B. Barbara, Phys. Rev. Lett. 1996, 77, 1873.

[28] U. Gysin, S. Rast, A. Aste, T. Speliotis, C. Werle, E. Meyer, Nanotechnology 2011, 22, 285715.

[29] P. Banerjee, F. Wolny, D. V. Pelekhov, M. R. Herman, K. C. Fong, U. Weissker, T. Mühl, Y. Obukhov, A. Leonhardt, B. Büchner, P. C. Hammel, Appl. Phys. Lett. 2010, $96,252505$. 


\section{WILEY-VCH}

[30] D. P. Weber, D. Rüffer, A. Buchter, F. Xue, E. Russo-Averchi, R. Huber, P. Berberich, J. Arbiol, A. Fontcuberta I Morral, D. Grundler, M. Poggio, Nano Lett. 2012, 12, 6139.

[31] B. Gross, D. P. Weber, D. Rüffer, A. Buchter, F. Heimbach, A. F. i Morral, D. Grundler, M. Poggio, 2015, 32.

[32] S. Lee, E. W. Moore, S. A. Hickman, J. G. Longenecker, J. A. Marohn, J. Appl. Phys. 2012, 111, 083911.

[33] Y. Tao, P. Navaretti, R. Hauert, U. Grob, M. Poggio, C. L. Degen, Nanotechnology n.d., $26,465501$.

[34] A. Kamra, M. Schreier, H. Huebl, S. T. B. Goennenwein, Phys. Rev. B 2014, 89, 184406.

[35] A. Buchter, J. Nagel, D. Rüffer, F. Xue, D. P. Weber, O. F. Kieler, T. Weimann, J. Kohlmann, a. B. Zorin, E. Russo-Averchi, R. Huber, P. Berberich, A. Fontcuberta i Morral, M. Kemmler, R. Kleiner, D. Koelle, D. Grundler, M. Poggio, Phys. Rev. Lett. 2013, 111, 067202.

[36] M. A. Zeeshan, S. Pané, S. K. Youn, E. Pellicer, S. Schuerle, J. Sort, S. Fusco, A. M. Lindo, H. G. Park, B. J. Nelson, Adv. Funct. Mater. 2013, 23, 823.

[37] S. Agramunt-Puig, N. Del-Valle, E. Pellicer, J. Zhang, J. Nogués, C. Navau, A. Sanchez, J. Sort, New J. Phys. 2016, 18, 013026.

[38] R. Hertel, J. Kirschner, J. Magn. Magn. Mater. 2004, 278, 291.

[39] U. Hartmann, Phys. Rev. B 1987, 36, 2331.

[40] O. Ergeneman, K. M. Sivaraman, S. Pané, E. Pellicer, a. Teleki, a. M. Hirt, M. D. Baró, B. J. Nelson, Electrochim. Acta 2011, 56, 1399.

[41] L. G. Vivas, M. Vazquez, J. Escrig, S. Allende, D. Altbir, D. C. Leitao, J. P. Araujo, Phys. Rev. B - Condens. Matter Mater. Phys. 2012, 85, 1.

[42] D. Kim, D. Y. Park, B. Y. Yoo, P. T. A. Sumodjo, N. V. Myung, Electrochim. Acta $\mathbf{2 0 0 3}, 48,819$. 


\section{WILEY-VCH}

[43] M. S. Viqueira, N. Bajales, S. E. Urreta, P. G. Bercoff, J. Appl. Phys. 2015, 117, 204302.

[44] D. Givord, M. E. Rossignol, D. W. Taylor, J. Phys. IV 1992, 2, C3.

[45] D. P. Weber, Dynamic Cantilever Magnetometry of Individual Ferromagnetic Nanotubes, University of Basel, 2014.

[46] C. Kittel, Introduction to Solid State Physics, 8th Edition, Wiley, 2005.

[47] L. Pauling, Phys. Rev. 1938, 54, 899.

[48] J. Chen, E. Flick, H. H. Gatzen, J. Appl. Phys. 2010, 107, 09A311.

[49] M. P. Raphael, J. A. Christodoulides, S. N. Qadri, B. S. Simpkins, J. M. Byers, Nanotechnology 2010, 21, 285101.

[50] M. Lévy, F. Lagarde, V.-A. Maraloiu, M.-G. Blanchin, F. Gendron, C. Wilhelm, F. Gazeau, Nanotechnology 2010, 21, 395103.

[51] U. Gneveckow, A. Jordan, R. Scholz, V. Brüss, N. Waldöfner, J. Ricke, A. Feussner, B. Hildebrandt, B. Rau, P. Wust, Med. Phys. 2004, 31, 1444.

[52] M. P. Kummer, J. J. Abbott, B. E. Kratochvil, R. Borer, A. Sengul, B. J. Nelson, IEEE Trans. Robot. 2010, 26, 1006.

[53] S. Erni, S. Schuerle, A. Fakhraee, B. E. Kratochvil, B. J. Nelson, J. Micro-Bio Robot. 2013, 8, 107.

[54] K. M. Pondman, N. D. Bunt, A. W. Maijenburg, R. J. A. Van Wezel, U. Kishore, L. Abelmann, J. E. Ten Elshof, B. Ten Haken, J. Magn. Magn. Mater. 2015, 380, 299. 


\section{WILEY-VCH}

(a)

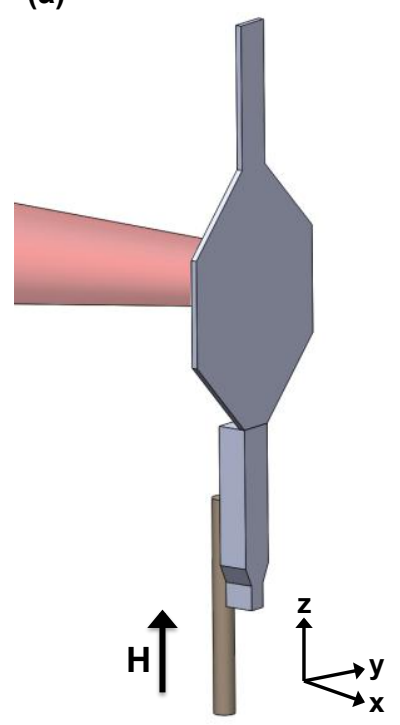

(b)

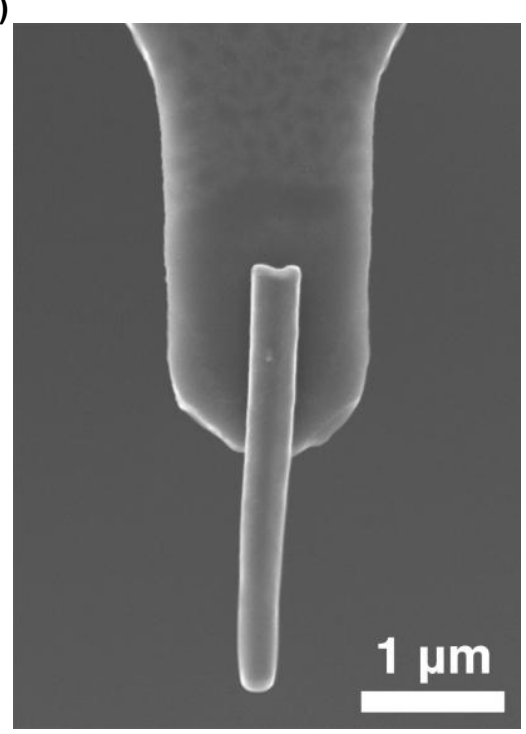

Figure 1. Single nanowire cantilever magnetometry. (a) Schematic illustration of the measurement technique. (b) A NiCo nanowire $\left(\mathrm{NW}_{1}\right)$ is attached to the cantilever tip for axial magnetization measurement.

(a)

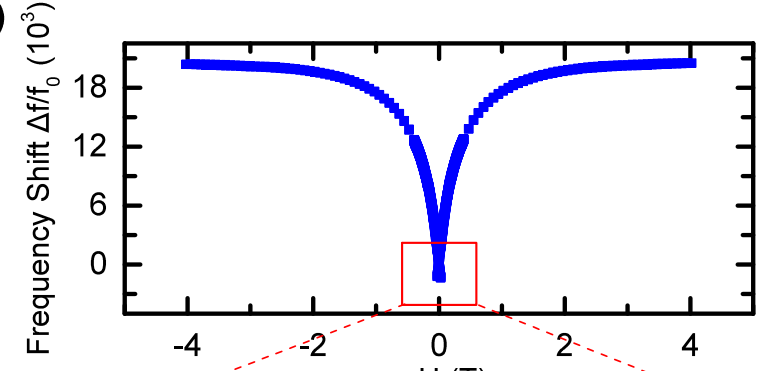

(b)

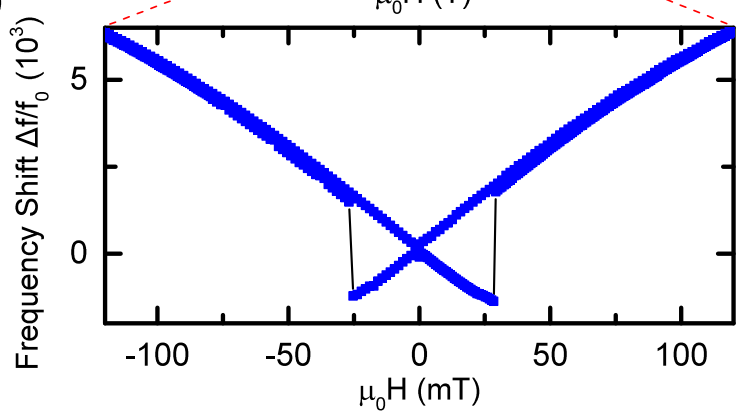

(c)

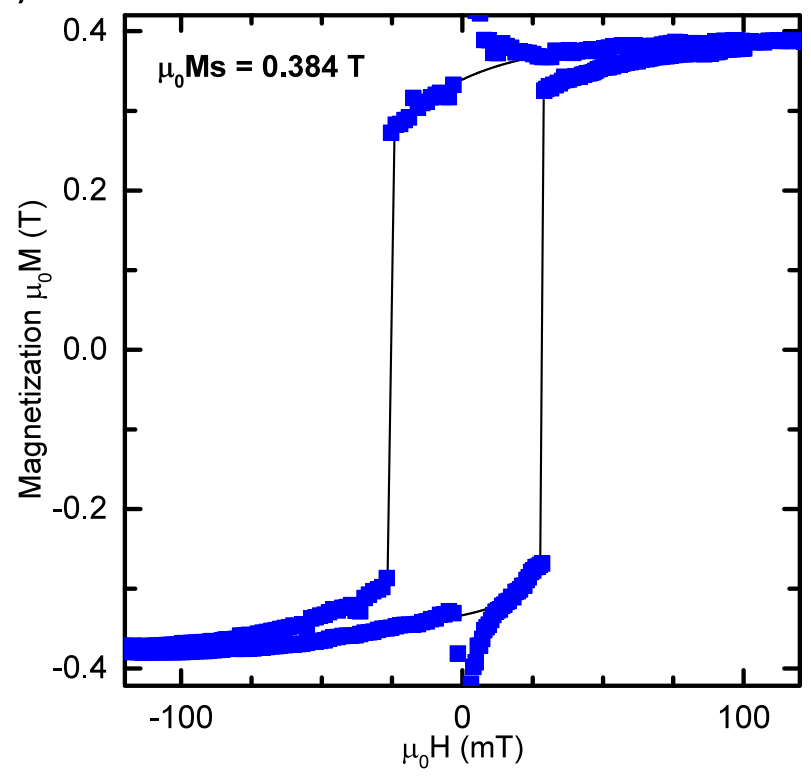

Figure 2. Cantilever magnetometry of single Ni nanowire (a) Resonant frequency shift as a function of applied magnetic field (b) Low-field $\left(\mu_{0} \mathrm{H}<50 \mathrm{mT}\right)$ frequency switching event (c) The corresponding magnetization loop of the Ni nanowire. Solid black lines guide the eye. 


\section{WILEY-VCH}

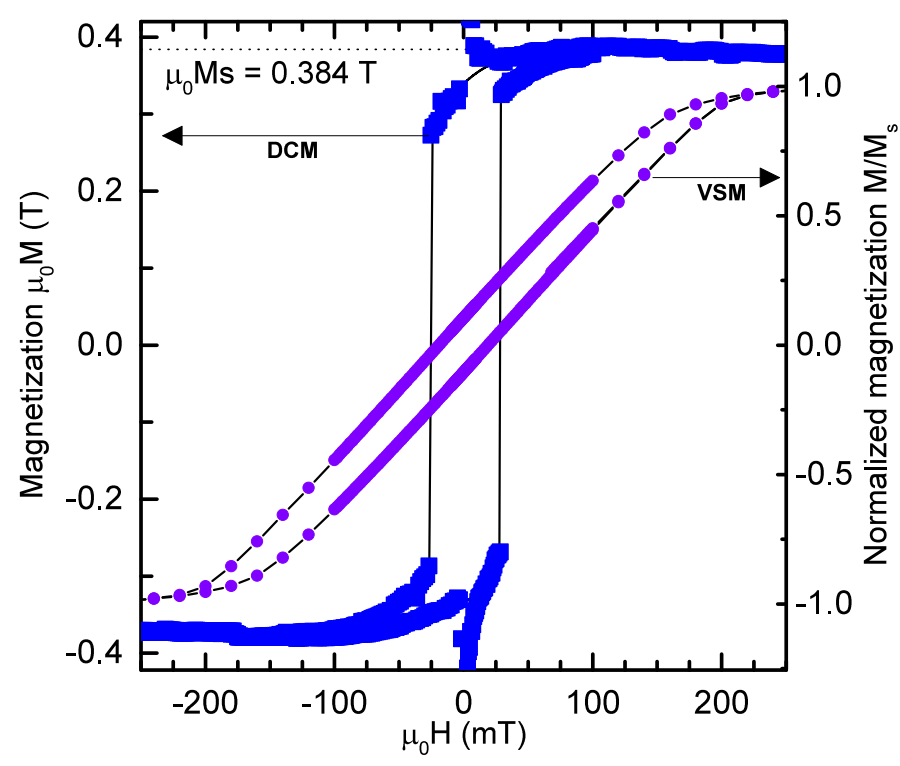

Figure 3. Single nanowire magnetometry compared to VSM measurements of the $\mathrm{Ni}$ nanowire array in the aluminum oxide templates (magnetic field applied parallel to the nanowire long axis). The bulk measurements show increased loop shear and low saturation remanence. Solid black lines guide the eye.
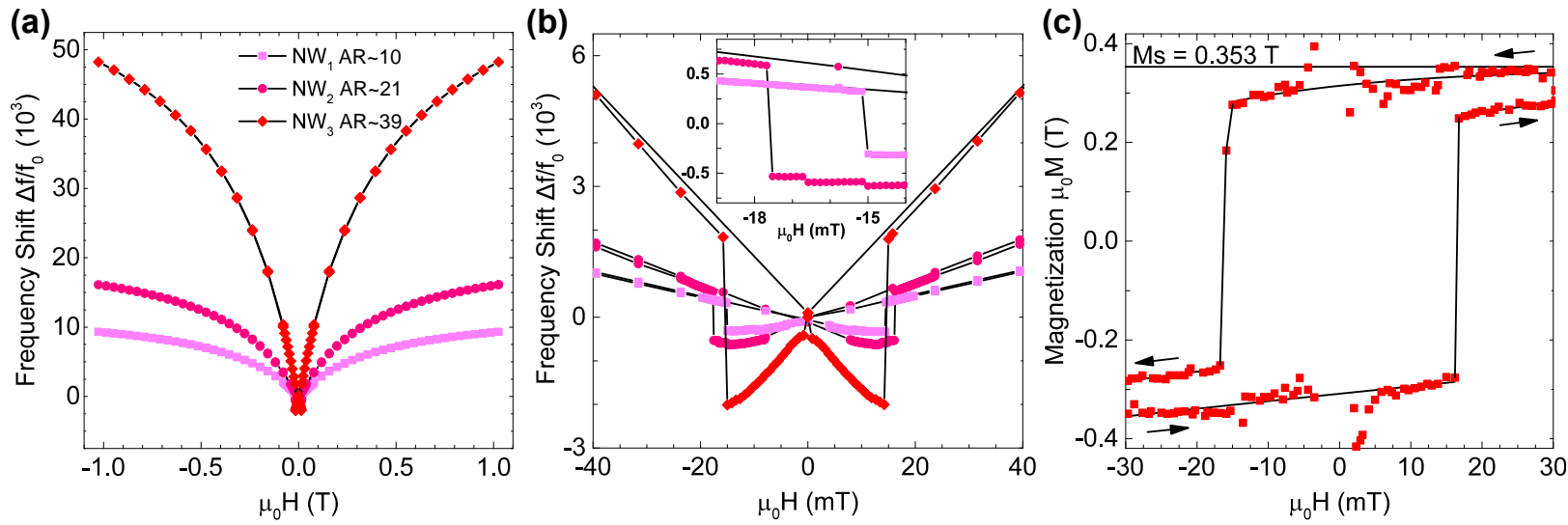

Figure 4. (a, b) Single nanowire magnetometry (SNM) was performed on $\mathrm{Ni}_{80} \mathrm{Co}_{20}$ nanowires of varying aspect ratio (AR). (c) A demonstrative example of the $\mathrm{M}-\mathrm{H}$ loop of an individual $\mathrm{Ni}_{80} \mathrm{Co}_{20}$ nanowire. Solid black lines guide the eye. 

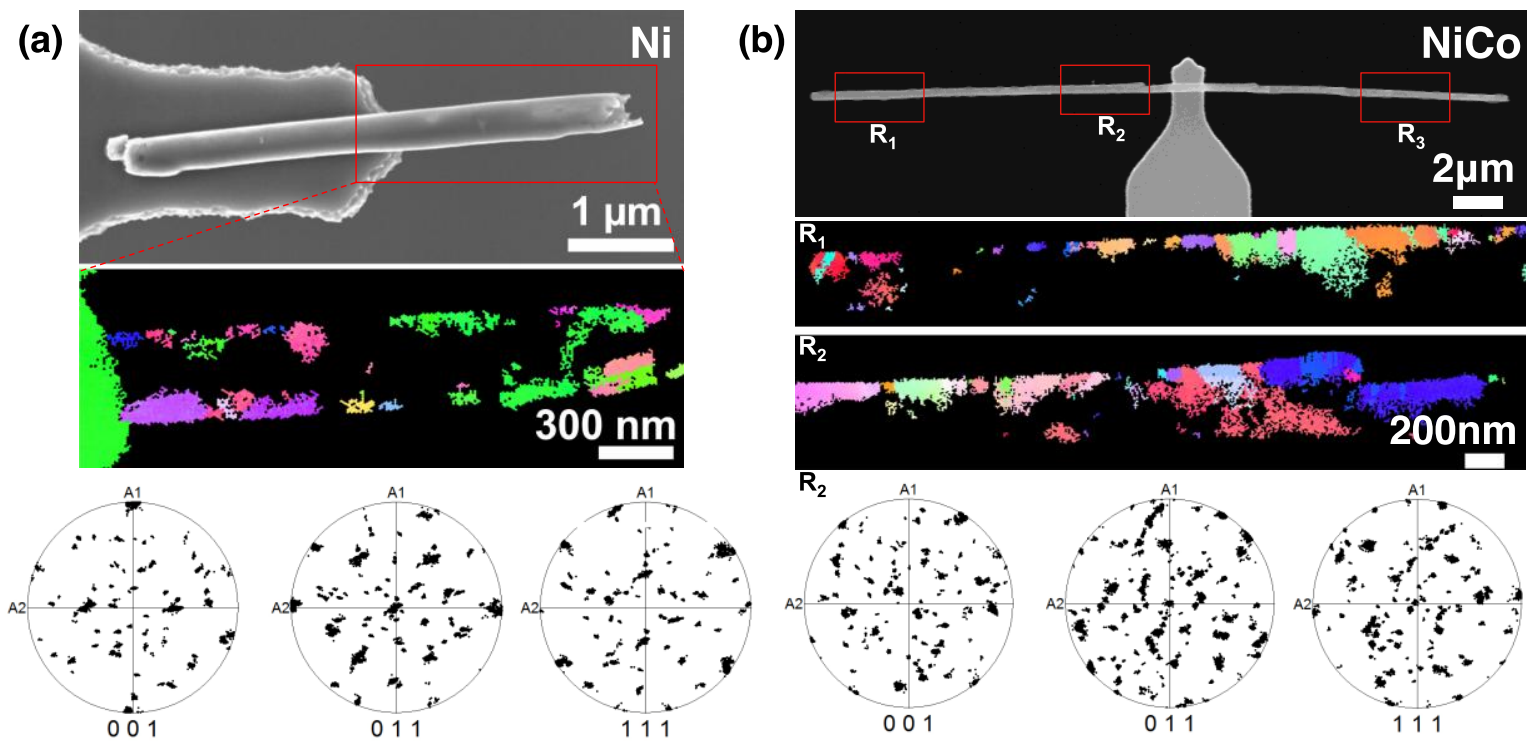

Figure 5. SEM and EBSD maps of the nanowires. (a,b) SEM images of the Ni and a representative $\mathrm{Ni}_{80} \mathrm{Co}_{20}$ taken right after cantilever magnetometry. The color mapping shows the distinct grains identified through EBSD in regions $\left(\mathrm{R}_{\mathrm{i}}\right)$, marked with a red box, while the adjacent sub-figure shows the spatial orientation of the crystallites in a pole figure. The curvature of the nanowires with respect to the detector leads to certain non-accessible regions as indicated by the black areas in the color-map. For the color-map to crystallographic orientation, see Figure S5.

\begin{tabular}{|c|c|c|c|c|c|}
\hline \multirow[t]{2}{*}{ Parameters } & & \multirow[t]{2}{*}{$\mathbf{N i}$} & \multicolumn{3}{|c|}{$\mathrm{Ni}_{80} \mathrm{Co}_{20}$} \\
\hline & & & $\mathrm{NW}_{1}$ & $\mathrm{NW}_{2}$ & $\mathrm{NW}_{3}$ \\
\hline \multirow[t]{2}{*}{ Geometry } & $\begin{array}{l}\text { Length } \\
(\mu \mathrm{m})\end{array}$ & 4.52 & 3.01 & 7.24 & 13.83 \\
\hline & $\begin{array}{l}\text { Average } \\
\text { Diameter } \\
(\mathrm{nm})\end{array}$ & 378 & 309 & 345 & 357 \\
\hline \multirow{9}{*}{$\begin{array}{l}\text { Cantilever } \\
\text { Magnetometry } \\
(\mathrm{T}=4 \mathrm{~K})\end{array}$} & $\begin{array}{l}m_{s} \\
\left(10^{-13} \mathrm{Am}^{2}\right)\end{array}$ & $1.547 \pm 0.15$ & $0.621 \pm 0.06$ & $1.407 \pm 0.14$ & $3.860 \pm 0.38$ \\
\hline & $\mu_{0} M_{S}(\mathrm{mT})$ & $384 \pm 94$ & $370 \pm 91$ & $264 \pm 65$ & $353 \pm 86$ \\
\hline & $\mu_{0} H_{c}(\mathrm{mT})$ & 29 & 14.4 & 16.2 & 15.8 \\
\hline & $\begin{array}{l}\mu_{0} H_{c+} \\
(\mathrm{mT})\end{array}$ & 305 & 300 & 180 & 256 \\
\hline & $\begin{array}{l}M\left(H_{C+}\right) \\
/ M_{s}\end{array}$ & $79.4 \%$ & $81.1 \%$ & $68.2 \%$ & $72.5 \%$ \\
\hline & $\mu_{0} M_{r}(\mathrm{mT})$ & 335 & 350 & 230 & 320 \\
\hline & $M_{r} / M_{s}$ & $87 \%$ & $95 \%$ & $87 \%$ & $91 \%$ \\
\hline & $\mu_{0} H_{s}(\mathrm{mT})$ & 70 & 53 & 118 & 91 \\
\hline & $\mu_{0} H_{k}(\mathrm{~T})$ & $0.328 \pm 0.03$ & $0.402 \pm 0.04$ & $0.398 \pm 0.04$ & $0.444 \pm 0.04$ \\
\hline
\end{tabular}


WILEY-VCH

\begin{tabular}{|c|c|c|c|}
\hline \multirow{3}{*}{$\begin{array}{l}\text { Vibrating } \\
\text { Sample } \\
\text { Magnetometry } \\
\text { (VSM) }\end{array}$} & $\begin{array}{l}\mu_{0} H_{c} \\
(\mathrm{~T}=103 \mathrm{~K})\end{array}$ & 20.95 & 10.70 \\
\hline & $\begin{array}{l}\mu_{0} H_{c} \\
(\mathrm{~T}=300 \mathrm{~K})\end{array}$ & 18.99 & 10.57 \\
\hline & $M_{r} / M_{S}$ & $10 \%$ & $2.9 \%$ \\
\hline
\end{tabular}

Table 1. Summary of magnetic measurements on single nanowires using DCM and bulk measurements using VSM. The corresponding nanowire geometries are also tabulated. The values are reported as mean $\pm \mathrm{SD}$.

This is the accepted version of the following article: Shamsudhin, N. et al. "Magnetometry of individual polycrystalline ferromagnetic nanowires" in Small, vol. 12, issue 46 (Dec. 2016), p. 6363-6369, which has been published in final form at DOI 10.1002/smll.201602338. This article may be used for noncommercial purposes in accordance with Wiley Terms and Conditions for Self-Archiving. 